\title{
Video recommendations for the Open Video project
}

\author{
Johan Bollen \\ Research Library \\ Los Alamos National \\ Laboratory \\ Los Alamos, NM \\ jbollen@lanl.gov
}

\author{
Michael L. Nelson and \\ Raquel Araujo \\ Department of Computer \\ Science \\ Old Dominion University \\ Norfolk, VA
}

\author{
Gary Geisler \\ Graduate School of Library \& \\ Information Science \\ Simmons College \\ Boston, MA 02115-5898 \\ gary.geisler@simmons.edu
}

\begin{abstract}
We describe a DL multimedia recommender system implemented for the Open Video project. Recommendations are generated by a spreading activation algorithm operating on a video network created from log download sequences. We compared the system's recommendations to those generated by a collaborative filtering technique.
\end{abstract}

\section{Categories and Subject Descriptors}

H.3.7 [Information Systems]: Digital Libraries

\section{General Terms}

Digital libraries, recommender systems, log data

\section{INTRODUCTION}

Although Collaborative Filtering (CF) algorithms [1] have shown to be effective tools for multimedia recommender systems, in a DL environment user behavior is often shaped by transitory information needs which invalidates the use of stored, long-term user profiles used in CF. Furthermore, many DLs can not practically or legally store user profiles. We propose a recommendation system that extracts document relationships from short-term co-download patterns recorded in a DL download log, and then generates spreading activation recommendations on the basis of the resulting networks. The Open Video project's, http: / / www . open-video. org, collection of videos was used as a test case for this approach.

\section{IMPLEMENTATION}

We analyzed 10,954 video downloads registered between July and November 2002 in the Open Video DL logs. Document titles were harvested for all 1320 downloaded videos. We extracted a set of video co-downloads from the mentioned log data, the latter defined as the same user downloading a pair of video with a small temporal latency. A co-download network of 1320 video relationships was generated. A Java Servlet was implemented to receive a

Permission to make digital or hard copies of all or part of this work for personal or classroom use is granted without fee provided that copies are not made or distributed for profit or commercial advantage and that copies bear this notice and the full citation on the first page. To copy otherwise, to republish, to post on servers or to redistribute to lists, requires prior specific permission and/or a fee.

JCDL 2005 Denver CO, USA

Copyright 200X ACM X-XXXXX-XX-X/XX/XX ...\$5.00. text query, map the query terms to video titles, and apply spreading activation to the video network. After the conclusion of this process, videos were ranked according to their final activation values and returned to the user. Table 1 lists the relationships generated for the video entitled "San Francisco Earthquake Aftermath, 1906".

\begin{tabular}{c|c}
\hline \multicolumn{2}{c}{ San Francisco Earthquake Aftermath, 1906 } \\
\hline weight & Video Title \\
\hline 0.96 & Hidden Fury, segment 09 of 11 \\
0.49 & Shock Troops of Disaster: Story of New England Hurricane \\
0.44 & S. F. Earthquake Aftermath: Riding Down Market St. \\
0.17 & Your Chance to Live: Earthquake \\
0.10 & San Francisco Earthquake Aftermath
\end{tabular}

Table 1: Relationships generated for "San Francisco Earthquake Aftermath, 1906" video

\section{EVALUATION}

We implemented a base-line CF recommendation system which derived user profiles from the above mentioned Open Video log. Item similarities were calculated on the basis of cosine similarities between the resulting user-item vectors [2]. We selected the top 10 downloaded videos and generated a set of relevant recommendations for each of these videos. Precision and recall scores were determined for $\mathrm{CF}$ and SA recommendation. The log data based SA outperformed the CF system by a wide margin in terms of its precision as well as its recall.

\section{Acknowledgements}

Rick Luce and Herbert Van de Sompel have supported and contributed to many of the ideas outlined in this paper. We also thank Somasekhar Vemulapalli and Weining Xu for their work on the systems presented here.

\section{REFERENCES}

[1] Jonathan L. Herlocker, Joseph A. Konstan, Al Borchers, and John Riedl. An algorithmic framework for performing collaborative filtering. In Proceedings on the 22nd Annual International ACM SIGIR Conference on Research and Development in Information Retrieval, pages 230-237, Berkeley, CA, August 15-19 1999.

[2] Badrul Sarwar, George Karypis, Joseph Konstan, and John Reidl. Item-based collaborative filtering recommendation algorithms. In Proceedings of the tenth international conference on World Wide Web, pages 285-295. ACM Press, 2001. 